normotensive pregnancies. Associations were stronger with pre-eclampsia than gestational hypertension, and increased with a greater number of affected pregnancies. Gestational hypertension was most strongly associated with strokes, in particular ischemic stroke, and pre-eclampsia was most strongly associated with acute myocardial infarction and cardiomyopathy.

Conflict of Interest None

\section{NON-SPECIFIC CHEST PAIN HOSPITAL ADMISSIONS AND READMISSIONS FOR SERIOUS CARDIOVASCULAR EVENTS IN THE UNITED STATES}

${ }^{1}$ Shing Kwok*, ${ }^{2}$ David Brown, ${ }^{3}$ Harriette Van Spall, ${ }^{4}$ Mary Norine Walsh, ${ }^{5}$ Aditya Bharadwaj, ${ }^{5}$ Purvi Parwani, ${ }^{6}$ Jessica Potts, ${ }^{7}$ Yoon Loke, ${ }^{8}$ Glen Martin, ${ }^{8}$ Evangelos Kontopantelis, ${ }^{9}$ David Fischman, ${ }^{6}$ Mamas Mamas. ${ }^{1}$ Royal Stoke University Hospital; ${ }^{2}$ Washington University School of Medicine; ${ }^{3}$ McMaster University; ${ }^{4}$ St Vincent Heart Center; ${ }^{5}$ Loma Linda University; ${ }^{6}$ Keele University; ${ }^{7}$ University of East Anglia; ${ }^{8}$ University of Manchester; ${ }^{9}$ Thomas Jefferson University Hospital

\subsection{6/heartjnl-2019-BCS.95}

Introduction Chest pain is one of the most common reasons for presentation to hospital. The objective of the evaluation of patients with chest pain is to identify acute coronary syndrome (ACS) and other serious cardiovascular conditions. However, diagnostics may not be $100 \%$ sensitive or specific, and important diagnoses may be missed during initial admission. No prior national study has evaluated readmission for serious cardiovascular events among the cohort of patients who are discharged with a diagnosis of non-specific chest pain.

Methods We conducted an analysis of patients with a primary diagnosis of non-specific chest pain in the Nationwide Readmission Database. We determined the rates, trends and predictors of readmission for serious cardiovascular events (ACS, pulmonary embolism (PE) and aortic dissection (AD)) after an inpatient episode. Multiple logistic regressions were used to identify predictors of readmission for ACS, PE and $\mathrm{AD}$ and the cost of the readmissions were determined.

Results A total of $1,172,430$ patients had an index diagnosis of non-specific chest pain between 2010 and 2014. Readmission for ACS, PE and AD were 2.4\%, $0.4 \%$ and $0.06 \%$, respectively within 6 months of discharge. Variables associated with ACS readmissions were diabetes (OR 1.49 95\%CI 1.171.32), coronary artery disease (OR 2.29 95\%CI 2.15-2.44), previous percutaneous coronary intervention (OR 1.65 95\%CI 1.56-1.75), previous coronary artery bypass graft (OR 1.52 95\%CI 1.43-1.61) and discharge against medical advice (OR 1.94 95\%CI 1.78-2.12). Female patients (OR 0.82 95\%CI $0.78-0.86)$ and patients in whom a coronary angiogram was undertaken (OR 0.48 95\%CI $0.45-0.52$ ) were less likely to have a readmission for ACS. Predictors of PE readmission were pulmonary circulatory disorder (OR 2.20 95\%CI 1.09 4.43), anaemia (OR 1.62 95\%CI 1.40-1.86) and cancer (OR 4.15 95\%CI 3.43-5.02). Predictors of AD readmissions were peripheral vascular disease (OR 8.63 95\%CI 5.47-13.60) and renal failure (OR 2.08 95\%CI 1.34-3.24). There were approximately 5,586 ACS readmissions, 950 pulmonary embolism readmissions and 113 aortic dissection readmissions in our study cohort and the estimated financial impact was $\$ 99$ million, $\$ 17$ million and $\$ 3.5$ million dollars of healthcare expenditure for ACS, PE and AD, respectively.

Conclusion Readmissions for serious cardiovascular disease following admission for non-specific chest pain are not uncommon and its financial burden can be significant accounting for approximately $\$ 120$ million dollars of healthcare expenditure in the United States between 2010 and 2014. Clinicians should be careful to appropriately investigate, risk stratify and tailor services to better manage patients with non-specific chest pain.

Conflict of Interest None

\section{HYPERTENSION OR HYPERTROPHIC CARDIOMYOPATHY? USING CARDIOVASCULAR MAGNETIC RESONANCE IMAGING TO UNMASK THE GREAT IMITATOR} ${ }^{2}$ Chris Miles, ${ }^{2}$ Rishi Patel, ${ }^{2}$ gemma parry-williams, ${ }^{2}$ Bode Ensam, ${ }^{2}$ Teck Khong Khong, ${ }^{2}$ Michael Papadakis, ${ }^{2}$ Maite Tome, ${ }^{2}$ Sanjay Sharma, ${ }^{3}$ Lisa Anderson. ${ }^{1}$ St. George's University of London; ${ }^{\text {sgul; }}{ }^{3}$ St Georges University Hospital

\subsection{6/heartjnl-2019-BCS.96}

Background Structural cardiac adaptations caused by hypertension present a diagnostic challenge when differentiating from hypertrophic cardiomyopathy (HCM), using traditional imaging techniques such as echocardiography (echo). Cardiac magnetic resonance imaging (CMR) offers reproducible anatomical, functional quantification as well as myocardial tissue characterisation which discriminates between hypertension and HCM.

Purpose To identify hypertensive individuals with undiagnosed HCM using CMR imaging.

Methods 100 consecutive African Caribbean (AC) and Caucasian hypertensive patients underwent $\mathrm{CMR}$ at a tertiary centre dedicated blood pressure clinic $(55 \%$ male, mean age 51 years). In keeping with ESC guidelines, end diastolic wall thickness (EDWT) $15 \mathrm{~mm}$ identified individuals within the "grey zone" between hypertension and with a potential HCM

Abstract 99 Table 1 p-values for CMR data in hypertensive (HTN), gray zone hypertensive (GZH) and hypertrophic cardiomyopathy (HCM) cohorts

\begin{tabular}{|l|l|l|l|}
\hline CMR Parameter & HCM vs, HTN & GZH vs. HTN & GZH vs. HCM \\
\hline EDVI & NS & $\mathrm{P}=0.025$ & NS \\
\hline EDWT & $\mathrm{P}=0.0002$ & $\mathrm{P}<0.0001$ & NS \\
\hline LVMI & $\mathrm{P}=0.0004$ & $\mathrm{P}<0.0001$ & NS \\
\hline
\end{tabular}

